\section{$\underset{\substack{\text { hommes } \\ \text { \& migrations }}}{ }$}

\section{Hommes \& migrations}

Revue française de référence sur les dynamiques

migratoires

$1326 \mid 2019$

Londres et ses migrations

\title{
«Londres ou rien » : rites de passage chez les jeunes Australiens de Londres
}

\section{Erica Consterdine et Michael Collyer}

\section{(2) OpenEdition \\ Journals}

Édition électronique

URL : https://journals.openedition.org/hommesmigrations/9665

DOI : 10.4000/hommesmigrations.9665

ISSN : 2262-3353

Éditeur

Musée national de l'histoire de l'immigration

Édition imprimée

Date de publication : 1 juillet 2019

Pagination : 59-67

ISBN : 978-2-919040-46-9

ISSN : $1142-852 X$

\section{Référence électronique}

Erica Consterdine et Michael Collyer, « «Londres ou rien » : rites de passage chez les jeunes Australiens de Londres », Hommes \& migrations [En ligne], 1326 | 2019, mis en ligne le 01 janvier 2022 consulté le 16 janvier 2022. URL : http://journals.openedition.org/hommesmigrations/9665 ; DOI : https://doi.org/10.4000/hommesmigrations.9665 


\section{«Londres ou rien»: rites de passage chez les jeunes Australiens de Londres}

\section{Erica Consterdine,}

chercheure associée au Sussex Centre for Migration Research (SCMR) et au départements de sciences politiques, université du Sussex,

\section{et Michael Collyer,}

professeur de géographie, université du Sussex.

Londres constitue une destination de choix pour la jeunesse australienne, première bénéficiaire du Youth Mobility Scheme (YMS). Ce programme de mobilité destiné aux ressortissants du Commonwealth permet aux jeunes migrants de certaines nations de travailler et de résider au Royaume-Uni pendant deux ans au maximum. Les jeunes Australiens sont nombreux à se retrouver dans cette capitale cosmopolite, concevant leur séjour londonien comme un véritable rite collectif de passage. Ce faisant, ils endossent le statut paradoxal de migrants temporaires qui se comportent dans leur ville d'accueil comme s'ils étaient chez eux.

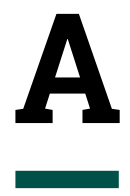

lors que le Royaume-Uni cherche à se détacher de l'Union européenne (UE), les questions sur l'avenir de Londres en tant que grand centre financier et urbain de l'Europe sont nombreuses. Londres a longtemps été considérée comme une «ville de grande diversité», très différente - tant sur le plan politique que démographique - du pays bien réel qu'elle représente. Londres est indéniablement une ville d'immigration: selon une étude réalisée par l'Observatoire des migrations en 2018, environ $41 \%$ de sa population est née hors du Royaume-Uni. Les migrants qui sont attirés par Londres contribuent à perpétuer ce cosmopolitisme si essentiel à son caractère. Ils sont attirés par le dynamisme urbain et les opportunités qu'offre cette ville en termes d'emploi. Plus que partout ailleurs au Royaume-Uni, la migration influence et nourrit tous les aspects de la vie à Londres.

Avec l'arrivée imminente du Brexit, le projet visant à mettre fin à la libre circulation soulève des questions majeures sur la diversité future de Londres, ainsi que sur les pénuries de main-d'œuvre qui en découleront. Les visas octroyés aux ressortissants de pays tiers qui en ont besoin se déclinent en cinq «niveaux», dont certains sont plus utilisés que 
d'autres. L'une des propositions du gouvernement pour répondre aux pénuries de main-d'œuvre après le Brexit a été d'étendre le "Tier 5 Youth Mobility Scheme» («Programme de mobilité Tier 5 destiné aux jeunes», YMS), qui permet aux jeunes migrants de certaines nations de travailler et de résider au Royaume-Uni pendant deux ans au maximum¹. Ainsi, 40864 «visas YMS » ont ainsi été délivrés en 2018, et ce sont les jeunes Australiens qui dominent dans ce système, avec $58 \%$ du total des visas YMS de niveau 5 attribués aux ressortissants australiens ${ }^{2}$. Néanmoins, en l'absence d'exigences de parrainage autour des visas YMS de niveau 5, on sait peu de chose sur la mobilité des jeunes Australiens. Nos recherches ont permis de combler certaines de ces lacunes.

La migration à Londres a une longue histoire, et les migrants issus du Commonwealth, surtout dans l'après-guerre, en font largement partie. Dans la Grande-Bretagne de l'après-guerre, la politique migratoire était dominée par un consensus bipartite visant à garantir aux 53 pays du Commonwealth un statut particulier. Les migrants issus de ce qu'on appelle l'«ancien Commonwealth», composé essentiellement du Canada, de l'Irlande, de l'Australie et de la Nouvelle-Zélande - et donc majoritairement blancs -, étaient privilégiés par rapport aux citoyens du Nouveau Commonwealth. Le projet du Commonwealth fut et demeure un vestige impérial fondé sur les idées de l'Empire, et le Youth Mobility Scheme en est le pur produit. Créé pendant la période de l'après-guerre en tant que concession aux citoyens du Commonwealth à la suite de l'adhésion du Royaume-Uni à la Communauté européenne et de la fin des privilèges du Commonwealth ${ }^{3}$, le Youth Mobility Scheme a longtemps été soutenu par la situation géopolitique.

Le présent article s'appuie sur des enquêtes et des entretiens réalisés avec de jeunes Australiens résidant à Londres dans le cadre du Youth Mobility scheme, dans le but de sonder ce qui les a poussés à migrer vers l'Angleterre. Nous avons constaté que les Australiens du YMS sont à mi-chemin entre le statut de travailleurs migrants et celui de touristes, attirés moins par le Royaume-Uni en tant que tel que par la capitale en particulier, migrant à Londres pour les possibilités de voyages en Europe qu'offre cette ville, ainsi que pour sa diversité cosmopolite à laquelle, d'ailleurs, ils contribuent. Contrairement à d'autres travailleurs migrants, cette décision de s'installer à Londres n'est pas motivée par des raisons économiques. Les Australiens du YMS entreprennent ce séjour comme un rite de passage, une période d'«auto-exploration» et d'individualisation avant de «devenir adultes» et de retourner en Australie.

Il s'agit là d'une forme privilégiée de mobilité opérant comme une forme de capital culturel au moment du retour dans le pays d'origine. Le principal paradoxe des jeunes Australiens est qu'ils se sentent profondément intégrés en Grande-Bretagne; bien qu'ils ne socialisent qu'avec d'autres Australiens une fois sur place, ils sont, de fait, des migrants temporaires qui se comportent comme s'ils étaient chez eux. Pour comprendre un tel paradoxe, il est essentiel d'analyser les fondements coloniaux du programme YMS et, par conséquent, le fait que ces jeunes Australiens soient blancs, ce qui leur confère une position privilégiée dans la «hiérarchie de l'immigration».

\section{La migration australienne à Londres}

L'Australie et le Royaume-Uni sont liés par un long passé de migration, favorisé par le cadre du Commonwealth qui a facilité cette mobilité entre les deux pays. L'Australie reste, en effet, la première destination des émigrants britanniques de longue durée. En 2017, 33 \% de tous les émigrants nés en Grande-Bretagne et vivant hors du Royaume-Uni résidaient en Australie ou en Nouvelle-Zélande ${ }^{4}$. Inversement, selon le recensement britannique de 2001, 107871 personnes nées en Australie résidaient au Royaume-Uni cette même année et, en 2016, l'ONS estimait que 125000 personnes nées en Australie résidaient au Royaume-Uni en 2015.

Tandis que l'immigration en provenance et à destination de l'Australie vers la Grande-Bretagne a été continuelle tout au long du XXe siècle, les schémas migratoires contemporains que nous observons aujourd'hui découlent des tendances et des développements qui ont eu lieu dans les années 1960, lorsque

1. UK Government, «The UK's Future Skills-based Immigration System », 2018. Url: https://www.gov.uk/government/ publications/the-uks-future-skills-based-immigration-system; Office national des statistiques, «Living Abroad: British Residents Living in the EU », avril 2018.

2. Home Office, «Why Do People Come to the UK? », 2018. Url: https://www.gov.uk/government/publications/immigrationstatistics-year-ending-september-2018/why-do-people-come-tothe-uk-2-to-work.

3. Erica Consterdine, « Community Versus Commonwealth: Reappraising the 1971 Immigration Act », in Immigrants \& Minorities, vol. 35, $n^{\circ} 1,2017$, pp. 1-20.

4. Office national des statistiques, op. cit. 
les baby boomers sont arrivés à l'âge adulte et que leur niveau de richesse générale a facilité les voyages. Les Australiens ont afflué en grand nombre à Londres dans les années 1960 et 1970, un phénomène symbolisé par ce qu'on a appelé la «colonisation de Earls Court», quartier qui allait même être surnommé par la suite «vallée des kangourous».

Alors que les prix des loyers ont grimpé en flèche dans le centre de Londres, la population australienne, tout comme l'ensemble des habitants de la ville, s'est dispersée géographiquement. Les lieux de résidence les plus populaires des Australiens à Londres sont désormais Shepherds' Bush, Fulham and Acton, Clapham, ainsi que Balham, Streatham, Tooting, Willesden, Kilburn et Queen's Park ${ }^{5}$. Si les personnes qui ont participé à notre étude étaient assez dispersées dans Londres, la majorité d'entre elles vivaient à Clapham $(n=17)$, Hammersmith et Fulham $(n=8)$.

\section{Le Commonwealth et la mobilité des jeunes}

Les citoyens des anciennes colonies ou du «vieux Commonwealth», dont l'Australie fait partie, bénéficient depuis longtemps d'un traitement privilégié au sein de la politique d'immigration du Royaume-Uni. Tandis que les migrations en provenance d'Asie du Sud et des Caraïbes ont suscité une hostilité massive de la part de l'opinion britannique d'après-guerre, "aucun mouvement politique ne s'est jamais mobilisé contre l'arrivée d'immigrants irlandais ou australiens, et ce malgré leur nombre souvent plus élevé et leur capacité vraisemblablement égale à rivaliser pour $s^{\prime}$ octroyer les ressources économiques ${ }^{6} »$. Randall Hansen a illustré la façon dont l'Empire et les héritages coloniaux ont tous deux mené à l'expansion initiale de la citoyenneté britannique sous la forme du «British Nationality Act» de 1948, qui a conféré des droits de citoyenneté britannique sans restriction à des centaines de millions de personnes vivant dans les anciennes colonies britanniques, entraînant à son tour un régime "restrictionniste» censé restreindre l'immigration «non blanche» issue du Commonwealth. Au cours des 33 années écoulées entre la loi de 1948 et l'abandon définitif de la citoyenneté du Commonwealth en vertu de la loi de 1981 sur la nationalité britannique, «les responsables politiques britanniques se sont engagés dans un effort plus ou moins explicite pour réformer ce cadre, afin de restreindre la migration issue des colonies non blanches, tout en maintenant les droits des citoyens des anciennes colonies blanches à la migration ${ }^{8}$. L'Empire, le Commonwealth et «l'édification de la nation» ont sans nul doute façonné l'expérience de l'immigration britannique en cette période d'après-guerre.

Historiquement, le programme britannique de mobilité des jeunes, anciennement connu sous le nom de «Working Holidaymakers Scheme» (WHM), est l'un des plus anciens programmes de ce type à avoir été mis en place dans le monde. À l'origine, le WHM était conçu comme un programme d'échanges culturels dédiés aux jeunes gens issus des pays du Commonwealth. Suite à l'introduction du système à points, en 2008, le WHM a été consolidé par d'autres programmes d'échanges temporaires (tel le «visa pour les jeunes hommes et jeunes filles au pair»). Rebaptisé "Youth Mobility Scheme» (YMS), il est subventionné au titre du «Tier 5 » pour la migration temporaire. Le changement le plus important apporté au régime a été le retrait des nouveaux pays du Commonwealth en tant que participants. Le YMS est actuellement limité à huit pays participants: l'Australie, le Canada, le Japon, Monaco, la Nouvelle-Zélande, Hong Kong, la République de Corée et Taïwan - pays auxquels est attribuée une allocation annuelle d'un nombre total de visas YMS sur la base des accords réciproques de visas YMS délivrés aux ressortissants britanniques sous les mêmes régimes. La liste des pays participants du programme est donc beaucoup plus restreinte que celle du programme WHM du Royaume-Uni, pour lequel tous les citoyens du Commonwealth étaient éligibles. Ce qui montre bien que l'effort porté sur la facilitation des échanges culturels entre citoyens du Commonwealth a été largement abandonné. Cela dit, on remarque que l'attribution de visas YMS aux anciens pays du Commonwealth est beaucoup plus importante que pour les pays n'appartenant pas au Commonwealth, la majorité étant attribuée aux Australiens (58 \% en 2018). Le visa YMS accorde une autorisation de séjour

\footnotetext{
5. Jude Wilson, David Fisher, Kevin Moore, «The OE Goes "Home": Cultural Aspects of a Working Holiday Experience », in Tourist Studies, vol. 9, n 1, 2009, p. 166.

6. Robert Ford, « Acceptable and Unacceptable Immigrants: How Opposition to Immigration in Britain Is Affected by Migrants' Region of Origin », in Journal of Ethnic and Migration Studies, vol. $37, n^{\circ} 7,2011$, p. 1020.

7. Randall Hansen, Citizenship and Immigration in Post-War Britain, Oxford, Oxford University Press, 2000.

8. Robert Ford, op. cit., p. 1021.
} 
de 24 mois et des restrictions de travail limitées, comprenant la non-obligation d'avoir un «employeur parrain » (et donc des qualifications reconnues), ainsi que la non-obligation de passer les tests habituels du marché du travail auxquels sont soumis les résidents étrangers.

Notre étude se donne pour but de sonder le paradoxe apparent des Australiens de Londres: s'ils bénéficient légalement des droits limités alloués aux migrants temporaires pour ce qui est de l'accès aux services publics et de la durée strictement limitée de leur séjour, ils ne s'en comportent pas moins comme s'ils étaient chez eux. Pour mener cette étude à bien, nous nous sommes appuyés sur les approches récentes de la "critical whiteness», qui détournent l'attention des études sur la race et l'ethnicité des groupes ethniques noirs, asiatiques et minoritaires (Bame) pour appréhender la «blancheur» comme une ethnicité à l'image des autres?.

\section{La migration comme rite de passage}

Si une attention considérable a été accordée, d'une part, à l'élite mondiale et, d'autre part, au mouvement et à la migration temporaires des ressortissants du «monde en développement» vers le monde industrialisé, les formes «intermédiaires» de migrants transnationaux ont été étonnamment peu prises en compte, "si l'on considère la notion d'"intermédiaire" par rapport à la situation socio-économique et de classe dans un pays d'origine ${ }^{10} »$. La mobilité des jeunes est à mi-chemin entre le tourisme et la migration liée à l'emploi, laquelle a longtemps représenté un rite de passage pour les jeunes migrants venant d'un pays lointain. Le volontarisme sous-tend ce type de migration, et la recherche du plaisir est l'élément qui distingue considérablement ce groupe de la migration économique ou des réfugiés politiques poussés par le besoin de s'exiler"11. Il n'existe à ce jour aucune recherche portant sur les migrants ayant bénéficié d'un programme de mobilité des jeunes au Royaume-Uni², ce qui s'explique par le fait que les migrants de «niveau 5 » restent politiquement cachés par leur association avec le tourisme.

En termes de parcours de vie et de transitions personnelles, l'élément «temps» est pertinent pour comprendre les motivations qui président à ce type de migration, car la majorité des jeunes Australiens vivent leur séjour à Londres comme un rite de passage. L'expérience culturelle, le tourisme et l'exploration individuelle sont les principales motivations de cette migration: «Il suffit de travailler pour pouvoir continuer à voyager» (participante âgée de 25 ans). Liée à la trajectoire de vie, la mobilité des jeunes est souvent considérée comme faisant partie intégrante de la transition de la jeunesse vers l'âge adulte.

La migration temporaire est perçue par les Australiens de l'YMS comme un rite de passage, un pas vers l'âge adulte, un processus «d'auto-expérimentation, d'exploration et de développement soutenus, permis par la perspective de début et de fin $q u^{\prime}$ offre le voyage ${ }^{13} \gg$. L'exploration de soi et la culture de l'individu ponctuent cette migration au cours de laquelle le voyage prolongé devient simplement une autre possibilité ouverte à l'individu: «Ce n'était pas pour le travail, ce n'était pas vraiment pour les vacances non plus. C'était plutôt lié à quelque chose comme la croissance personnelle... Un rite de passage et la chance de quitter l'Australie pour découvrir un endroit confortable, où il est facile de s'installer. Je pense qu'une partie de moi avait besoin de partir en exploration. Beaucoup d'amies allaient s'installer à Perth, vous voyez, elles étaient en couple depuis

9. Cet article se fonde sur 75 enquêtes et 20 entretiens approfondis réalisés auprès d'Australiens résidant au RoyaumeUni grâce à un visa octroyé par le biais du YMS Tier 5. Les participants ont été sélectionnés au moyen d'un échantillonnage en «boule de neige » et avec l'aide d'un groupe d'expatriés («Aussies in London», https://www.facebook.com/ AussielnLondon/) mis en place spécifiquement pour les jeunes Australiens de Londres. Nous avons mené une enquête confidentielle auprès de 75 migrants lors de divers événements organisés par le groupe, et 20 d'entre eux se sont portés volontaires pour des entretiens approfondis menés ultérieurement dans différents endroits de Londres entre avril 2016 et mai 2017. Ces entretiens ont été codés manuellement à l'aide d'un programme de codage de base afin d'identifier les thèmes clés ressortant de nos discussions, tout particulièrement les motivations qui les ont poussés à émigrer, les questions liées à l'intégration, la socialisation et leurs rapports au marché du travail.

10. David Conradson, Alan Latham, «Friendship, Networks and Transnationality in a World City: Antipodean Transmigrants in London », in Journal of Ethnic and Migration Studies, vol. 31, $n^{\circ} 2$, 2005, p. 290.

11. Raoul V. Bianchi, «Migrant Tourist-Workers: Exploring the "Contact Zones" of Post-Industrial Tourism », in Current Issues in Tourism, vol. 3, n², 2000, p. 125.

12. Voir les travaux suivants qui constituent les seules exceptions : Erica Consterdine, "Youth Mobility Scheme: The Panacea for Ending Free Movement? », in National Institute Economic Review, vol. 248, nº 1, 2019, pp. 40-48; David Conradson, Alan Latham, op. cit; Elsa T. Oommen, «Privilege and Youth Migration: Polarised Employment Patterns of Youth Mobility Workers in London », in Journal of Ethnic and Migration Studies, 2019, pp. 1-17.

13. David Conradson, Alan Latham, op. cit., p. 290. 
longtemps, elles se mariaient, l'une d'elles était enceinte. C'est simplement que je n'étais pas encore prête à franchir ce pas, alors l'idée de déménager dans une autre ville me semblait exaltante, et comme je suis quelqu'un d'assez angoissé, je me suis dit que si j'arrivais à le faire, je serais vraiment fière de moi... J'avais l'impression qu'il fallait que ce soit derrière moi avant d'entrer dans la trentaine» (participante âgée de 25 ans).

Pour beaucoup, l'expérience de l'étranger est un processus de réalisation de soi, et cette expérience en est venue à incarner des idéaux d'autonomie personnelle, de débrouillardise, d'aventure et d'expansion personnelle ${ }^{14}$. Toutes les personnes interrogées ont parlé de l'utilisation du visa YMS comme d'une «dernière chance» pour s'explorer et "grandir» avant de "s'installer». Cette idée d'une horloge temporelle liée au parcours de vie se retrouve dans l'exigence d'âge limite d'admissibilité du programme, puisque les participants ne peuvent être âgés de plus de 30 ans ou avoir des personnes à charge. Ce qui contribue à renforcer le rapport à la temporalité des jeunes Australiens et leur sentiment d'une «dernière chance »: «À cause de cette notion de "timing" [liée à l'obtention du visa YMS], on a l'impression qu'un sablier est en train de se vider: il faut y aller avant qu'il ne soit trop tard, c'est une occasion qui ne se reproduira pas. Comme vous êtes plus jeune, que vous n'êtes pas encore bien loin dans l'existence, l'investissement immobilier ou le fait d'avoir des enfants compte moins, on a moins besoin d'avoir des connaissances de base. Cela s'adapte mieux à la période de la vingtaine, quand vous venez peut-être de terminer vos études et que vous êtes intéressé par d'autres expériences» (participant âgé de 27 ans).

Cette installation temporaire en GrandeBretagne est considérée comme un pas vers l'âge adulte, une démonstration et un test d'indépendance et d'endurance pour ces Australiens jeunes et parfois nostalgiques, ce qui souligne à quel point cette mobilité est liée aux étapes de la transition: "I'ai l'impression que ce visa tient presque de l'horloge biologique, je voulais m'en servir avant de devenir un

14. Ibid., p. 299. 
peu plus vieux et un peu plus investi, que ce soit en matière de propriété ou dans une relation » (participant âgé de 28 ans). «D'une certaine manière, je pense qu'il [le visa YMS] répond à cette étape de notre vie, c'est une bonne motivation pour voyager, faire l'expérience d'un ailleurs et rentrer en Australie avec les expériences qu'on a glanées» (participant âgé de 29 ans).

L'un des aspects clés de ce rite de passage est l'idée que le fait de "déménager dans un logement» renforce la tolérance et prépare les individus à l'âge adulte, de sorte que ces «travailleurs saisonniers» rentrent chez eux avec des récits forts, confiants dans leur capacité à faire face au changement et « requalifiés pour un monde épisodique et fragmentaire, préparés aux tensions de la vie moderne (et ce malgré leur but qui, dans de nombreux cas, est d'échapper temporairement à ces mêmes tensions) ${ }^{15}$ ". "Vivre un tel changement m'a permis de véritablement croire en moi. J'ai gagné en confiance» (participante âgée de 26 ans).

Les Australiens se servent de la migration essentiellement comme d'un remodelage fluide de leur moi à chaque étape de leur vie. Les jeunes Australiens sont attirés par Londres en raison du style de vie et des expériences personnelles qu'ils peuvent y trouver, plutôt que du simple appât du gain. Pour les Australiens bénéficiant du YMS, leur motivation à émigrer temporairement à Londres réside ainsi dans ce remodelage et ce rite de passage, les considérations relatives au marché du travail restant secondaires.

\section{Capital culturel et «super-diversité »}

Ce qui est frappant à propos de ce type spécifique de mobilité, c'est que les jeunes Australiens ne décident pas d'émigrer spécifiquement en Grande-Bretagne, mais bien vers Londres, tout particulièrement en raison du style de vie et des possibilités de voyage qu'elle leur offre. La majorité des personnes interrogées ont déclaré qu'elles n'avaient pas envisagé et n'envisageraient pas de déménager dans une autre ville du Royaume-Uni, et que si l'option de Londres ne s'était pas présentée, elles n'auraient pas émigré au Royaume-Uni. En fait, très peu de personnes interrogées ont quitté Londres pendant leur séjour au Royaume-Uni. Ce n'est évidemment pas propre aux jeunes Australiens, et Londres n'a rien d'unique par rapport à d'autres villes mondiales qualifiées de «super-diverses». «Quand vous arrivez au RoyaumeUni, vous allez à Londres - c'est la plaque tournante.
Ce sera Londres ou rien » (participant âgé de 25 ans). «Au moment d'effectuer ma demande de départ depuis Perth, c'est très clairement Londres et elle seule qui m'a attiré... Pour être honnête, je ne m'intéressais à aucune autre ville» (participant âgé de 27 ans).

Les personnes interrogées racontent que Londres a «illuminé leur vie» en leur permettant de faire l'expérience de la "super-diversité»; c'était surtout le cas des personnes ayant grandi dans des villes rurales d'Australie: "On dit que Londres est une ville multiculturelle. Et on se dit: tu m'étonnes, c'est une des plus grandes villes qui existent, et puis, c'est en Europe. Mais, dans ma tête, je ne m'attendais pas à ce qu'elle soit aussi multiculturelle. Je m'étais imaginé à quoi pouvait ressembler une journée normale à Londres, et je n'aurais jamais imaginé tant de variété culturelle, surtout venant d'Australie où on est peutêtre multiculturels, mais encore sacrément blancs. Par exemple, quel Australien a déjà vu un Juif orthodoxe! Ça a vraiment élargi nos idées en matière de religion et de diversité culturelle» (participante âgée de 28 ans, participant âgé de 26 ans).

L'attrait de Londres réside principalement dans ses possibilités de transport global vers l'Europe et, plus généralement, dans sa nature de «plaques tournante» des flux de communication. 65 participants de notre étude ont voyagé en Europe pendant leur séjour en Grande-Bretagne, et 42 d'entre eux ont cité les voyages en Europe comme leur principale motivation pour venir s'installer à Londres. Les Australiens bénéficiant du YMS se sont rendus dans de multiples destinations européennes, passant souvent la majeure partie de leur temps libre en week-end dans d'autres villes. Ce sont précisément ces voyages de loisir à long terme qui, intégrés à l'activité professionnelle, contribuent à «étendre les dimensions à la fois temporelle et expérimentale du séjour outre-mer au-delà de ce que l'on entend communément par tourisme ${ }^{16}$ ». Et pourtant, cette mobilité est sans nul doute motivée - au moins en partie, sinon principalement - par le tourisme au sens large: "Savoir qu'on peut sauter dans un Eurostar et rejoindre Paris en claquant des doigts, ou prendre un avion quand ça nous chante, c'est quand même tentant» (participant âgé de 27 ans).

15. Nick Clarke, « Mobility, Fixity, Agency: Australia's Working Holiday Programme », in Population, Space and Place, vol. 10, $n^{\circ} 5$, 2004, p. 412.

16. Jude Wilson, David Fisher, Kevin Moore, op. cit., p. 160. 
Le tourisme est un moyen essentiel de formation de l'«identité cosmopolite», impliquant l'exploration d'espaces et des périodes de construction de soin. Ce remodelage du moi, qui fait partie intégrante de la migration des jeunes favorisée par les programmes de mobilité, constitue une tentative de "glaner un capital culturel que l'on pourra déployer lorsqu'on rentrera chez soilis》. Vivre à Londres est ainsi considéré comme une forme de capital culturel. Nombre de jeunes Australiens construisent leurs expériences de voyage comme une forme de capital leur permettant ensuite de démontrer leur cosmopolitisme: "Pour être honnête, il y a un peu de vantardise à dire que je vis à Londres alors que mes amis sont coincés à Perth» (participante âgée de 26 ans).

Mais il s'agit d'une migration privilégiée qui n'est accessible qu'aux personnes qui ont la souplesse nécessaire pour voyager à cette époque de leur vie, qui peuvent se permettre le coût élevé de la vie à Londres et accepter les emplois précaires qu'offre la capitale. Quantité de personnes interrogées ont parlé de leur migration comme d'une migration ponctuelle, une chance de pouvoir partir à l'aventure avant de s'installer définitivement dans leur pays d'origine. Il s'agit d'une forme de capital culturel en vertu duquel le fait de travailler à Londres peut faciliter les carrières en Australie, mais c'est surtout l'expérience même de vivre à Londres qui a valeur de statut social.

\section{L'intégration des migrants temporaires}

Les migrants temporaires sont par définition assujettis à une limitation temporelle; par conséquent, on imagine toujours que les migrants temporaires ne réussiront jamais à s'intégrer. Or on constate que les Australiens qui participent à l'YMS remettent en question ces idées reçues, car la majorité d'entre eux éprouvent un fort sentiment d'appartenance, certains ayant même l'intention de rester en Angleterre et utilisant I'YMS comme terrain d'essai transitoire. Les trois quarts des participants à notre enquête ont déclaré qu'ils se sentaient intégrés et éprouvaient un sentiment d'appartenance à la Grande-Bretagne, en dépit du fait qu'ils y vivaient depuis moins de deux ans. C'est là que réside le paradoxe des Australiens de Londres: il s'agit bien de migrants temporaires qui se comportent comme s'ils étaient chez eux. "Je me sens plus à l'aise, je me sens chez moi dans les pays du Commonwealth. Des gouvernements similaires appellent des modes de vie similaires, des lois similaires, des transports similaires. Je me sens mieux ici» (participante âgée de 28 ans).
Il est étonnant de constater à quel point les jeunes Australiens «temporaires» sont intégrés en Grande-Bretagne, tout en restant cantonnés au sein de leur propre diaspora; un phénomène qui s'explique par leur langue, leur culture, leur conformité ethnoculturelle, et enfin par les liens coloniaux historiques unissant les deux nations. Cette «culture commune» découle du projet du Commonwealth et, en fin de compte, du fait que les migrants blancs sont privilégiés par rapport aux migrants non blancs.

La «blancheur» est un élément clé permettant d'expliquer ce paradoxe. Comme toute ethnie, la «blancheur» est construite. Elle n'est pas directement liée aux caractéristiques biologiques héréditaires, mais à l'apparence et à la présentation ${ }^{19}$. Elle recoupe également d'autres vecteurs du pouvoir, tels que en particulier le genre, la classe ou la sexualité. Le principal progrès théorique apporté par les études critiques sur la «blancheur» aux études sur la race et l'ethnicité concerne l'accent mis sur la «blancheur» non pas comme absence d'ethnicité, comme la norme à laquelle toutes les autres ethnies font référence, mais comme une ethnie parmi tant d'autres. La blancheur comporte certains privilèges qui figurent l'envers du racisme structurel de sociétés comme le Royaume-Uni. Le fait d'être perçu comme blanc facilite les rencontres sociales et institutionnelles les plus importantes. Par conséquent, on s'attend tout bonnement à ce que les expériences bureaucratiques se déroulent plus facilement. De la même manière, cela entraîne un sentiment d'habilitation qui est essentiel à la compréhension de l'intégration. Le simple fait d'être blanc facilite le passage des Australiens du YMS dans la société britannique, même par rapport aux ressortissants de pays majoritairement non blancs parlant couramment l'anglais.

Si les jeunes Australiens ont l'impression de s'intégrer à Londres, très peu d'entre eux nouent des liens sociaux avec des Britanniques, et la majorité des participants et des personnes interrogées échangent exclusivement avec d'autres Australiens. En effet, le schéma des jeunes Australiens est le suivant: ils ont beau se sentir intégrés, ils auront tendance à ne socialiser qu'avec leurs compatriotes. "Je dis que je

17. Adrian Franklin, Tourism: An Introduction, New York, Sage Publications, 2003.

18. David Conradson, Alan Latham, op. cit., p. 292.

19. Annop Nayak, "Critical Whiteness Studies », in Sociology Compass, vol. 1, n², 2007, pp. 737-755 
me sens intégrée, mais quand j'y pense, je constate que nous socialisons seulement avec des Australiens. Alors, à quel point sommes-nous vraiment intégrés? Je vis ici, je travaille ici, je communique avec différentes cultures, mais en fin de compte, mes amis ne sont que des Australiens... Nous ne sommes pas si intégrés que ça» (participante âgée de 25 ans).

55 participants ont déclaré qu'ils communiquaient avec d'autres Australiens chaque jour ou chaque semaine à Londres, et 59 autres ont déclaré qu'ils connaissaient d'autres Australiens vivant à Londres avant leur arrivée. La majorité des participants ont vécu avec d'autres Australiens, et un certain nombre d'entre eux ont indiqué qu'ils avaient cherché d'autres Australiens avec qui vivre: "Avant, on vivait avec des Italiens, des Français, des Anglais... Ça n'est pas qu'on n'aimait pas ça, mais on a découvert qu'on a de meilleures expériences avec les Australiens et les "Kiwis" [Néo-Zélandais], on est là pour la même raison, on est là pour deux ans et on a les mêmes centres d'intérêt... C'est simplement qu'ils ne sont pas aussi ouverts que les Australiens pour faire de nouvelles rencontres, on est tous là pour se faire de nouveaux amis et rencontrer des gens différents, on aime voyager ensemble... J'ai l'impression de les connaitre, ils me rappellent la maison, on a le même sens de l'humour, on a plus en commun, et puis, on en est à la même étape de notre vie» (participante âgée de 23 ans).

\section{(1) La migration à Londres dans le cadre de l'YMS est une mobilité privilégiée, utilisée comme un rite de passage et une chance de voyager en Europe.}

Bien que le fait de vivre et d'échanger presque exclusivement avec d'autres Australiens n'était pas nécessairement une stratégie consciente, de nombreux participants estimaient qu'ils s'entendaient mieux avec d'autres Australiens. "Je n'avais pas l'intention de me lier d'amitié avec des Australiens, mais c'est arrivé de façon naturelle; il faut dire que nous avons le même sens de l'humour, la même éducation, les mêmes expériences. Je m'entends bien avec mes collègues de travail, et ils me trouvent marrante, mais ma vie sociale se déroule principalement avec des Australiens. J'ai l'impression qu'on a les mêmes opinions, et le sens de l'humour est vraiment déterminant. J'ai souvent l'air sarcastique, ça peut paraître impoli, mais les Australiens comprennent, eux» (participante âgée de 25 ans).

Ces témoignages révèlent une tension au sein de la jeune diaspora australienne, un tiraillement entre l'expérience du pays d'accueil et le besoin d'appartenance et d'identité collective. Certaines personnes interrogées ont parlé de la frustration qu'elles éprouvaient en raison de leurs échanges sociaux limités aux seuls Australiens et de leurs difficultés à nouer des relations avec les Anglais: "J'aimerais avoir plus d'amis anglais. C'est drôle: la plupart des amis que je me suis faits depuis que je suis ici sont des gens que je connaissais vaguement chez moi, et depuis que je vis ici, on est devenus plus proches. Parce qu'on a ça en commun: être loin de la maison» (participante âgée de 26 ans). Néanmoins, le besoin d'appartenance, l'orientation vers leur terre d'origine et leur besoin d'identité collective poussent de nombreux jeunes Australiens à rechercher des liens avec d'autres Australiens pendant leur séjour au Royaume-Uni.

\section{Conclusion}

Les Australiens vivant à Londres grâce à l'YMS remettent en question un certain nombre d'idées reçues quant à la migration temporaire - en particulier le paradoxe selon lequel les migrants temporaires auraient le sentiment de s'intégrer à un pays tout en n'y fréquentant que leurs compatriotes nationaux. Cette migration n'est motivée ni par la pauvreté, ni uniquement par le profit économique. Les migrants bénéficiant de l'YMS sont plutôt des individus se trouvant à mi-chemin entre les travailleurs migrants et les touristes, ce qui rend l'étude de leur «temporarité » d'autant plus singulière. La migration à Londres dans le cadre de l'YMS est une mobilité privilégiée, utilisée comme un rite de passage et une chance de voyager en Europe. Les Australiens ne sont pas attirés par la Grande-Bretagne mais bien par Londres en particulier, et, curieusement, par les possibilités de voyage que cette ville offre à l'extérieur de la Grande-Bretagne.

Même s'ils ne font qu'échanger avec leurs compatriotes, ces jeunes Australiens ressentent un sentiment d'appartenance à Londres. Un sentiment qui trouve sa source dans le projet du Commonwealth, le néocolonialisme et, en fin de compte, le privilège de la «blancheur». Si ce point de vue ne saurait être une critique du désir d'«aventure» exprimé par bon nombre des personnes interrogées, il n'en conduit pas moins à une reconnaissance de la nature du privilège 
dont ils sont nombreux à jouir. Cette soif d'aventures est partagée par beaucoup d'autres jeunes migrants, y compris ceux qui se retrouvent sans papiers à Londres ${ }^{20}$. La facilité et le confort de vie dont jouissent les jeunes Australiens qui ont participé à cette étude sont tout à leur honneur. Ils contribuent ainsi au riche mélange cosmopolite de Londres, tout comme d'autres migrants. Cependant, contrairement à d'autres migrants, ce paradoxe de la séparation mêlée à la participation exige des explications particulières.

Il est évident que ces explications peuvent être spécifiques aux Australiens, et peu d'éléments dans cette étude suggèrent que les attraits de l'YMS seront faciles à maintenir dans l'ère post-Brexit. Alors que le gouvernement et l'industrie cherchent des solutions à une crise du travail qui s'annonce imminente dans le cas où la libre circulation prendrait fin, l'extension de l'YMS aux pays européens a été présentée comme une solution possible. Nos résultats prouvent néanmoins que les Australiens bénéficiant de l'YMS constituent un groupe privilégié qui n'émigre pas au Royaume-Uni pour y travailler, mais bien pour voyager et faire de nouvelles expériences. Le travail est sans nul doute une considération secondaire. La clé du succès et de l'attrait du visa YMS est d'offrir aux migrants non ressortissants de l'UE la possibilité de voyager plus largement en Europe. C'est la raison essentielle pour laquelle les migrants australiens bénéficiant de l'YMS élisent le Royaume-Uni comme destination de choix. Étant donné que les citoyens de l'UE peuvent voyager, travailler et résider librement dans tous les États membres, il y a peu de raisons de penser que le Royaume-Uni demeurerait à cet égard attrayant pour les jeunes citoyens de l'UE.

20. Alice Bloch, Nando Sigona, Roger Zetter, « Migration Routes and Strategies of Young Undocumented Migrants in England: A Qualitative Perspective », in Alice Bloch, Milena Chimienti (dir.), Irregular Migrants: Policy, Politics, Motives and Everyday Lives, Londres, Routledge, 2013, pp. 26-42. 